\title{
Role of diaphragmatic rapid shallow breathing index in predicting weaning outcome in patients with acute exacerbation of COPD
}

This article was published in the following Dove Press journal: International Journal of COPD

\author{
Ahmad Abbas \\ Sameh Embarak \\ Mohammad Walaa \\ Samah Mohamed Lutfy \\ Chest Department, Faculty of \\ Medicine, Zagazig University, \\ Zagazig, Egypt
}

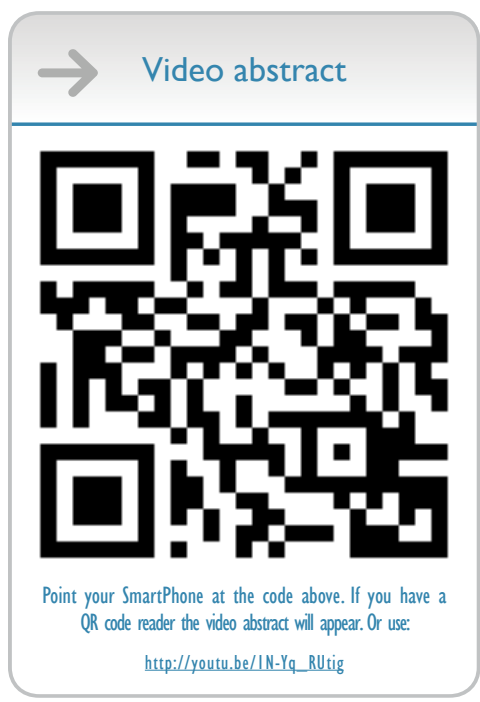

Correspondence: Ahmad Abbas Chest Department, Faculty of Medicine, Zagazig University, Port Said Street, Mit-Ghamr, 356I I, Egypt Email amabbaas@zu.edu.eg
Background: The diaphragmatic rapid shallow breathing index (D-RSBI), which is the ratio between respiratory rate $(\mathrm{RR})$ and the ultrasonographic evaluation of diaphragmatic displacement (DD), is a new and promising tool to predict weaning outcome. Its accuracy in predicting weaning failure, in ready-to-wean acute exacerbation COPD (AECOPD) patients, needs to be evaluated.

Patients and methods: A prospective observational study was carried out on ready-to-wean AECOPD patients. During a T-tube spontaneous breathing trial (SBT) evaluation of the right hemidiaphragm displacement (ie, DD), M-mode ultrasonography to calculate the D-RSBI, as well as the RSBI (RR/tidal volume [VT]) were carried out simultaneously. Outcome of the weaning trial was recorded. Receiver operating characteristic (ROC) curves were used to evaluate the diagnostic accuracy of D-RSBI and RSBI.

Results: A total of 50 AECOPD patients requiring mechanical ventilation for more than $48 \mathrm{~h}$ who were ready to perform a SBT were included. Of these, 37 (74\%) were successfully liberated from mechanical ventilation. Among the 13 patients who failed the weaning trial, $8(62 \%)$ failed the SBT and reconnected to the ventilator, 2 (15\%) were reintubated within $48 \mathrm{~h}$ of extubation and $3(23 \%)$ required NIV support within $48 \mathrm{~h}$ of extubation. The areas under the ROC curves for D-RSBI and RSBI were $0.97(p<0.001)$ and $0.67(p<0.06)$, respectively.

Conclusion: D-RSBI (RR/DD) is superior to the traditional RSBI (RR/VT) in predicting weaning outcome in AECOPD patients.

Keywords: rapid shallow breathing, diaphragmatic displacement, ultrasonography, spontaneous breathing trial, weaning

\section{Introduction}

Acute exacerbations of COPD (AECOPD) are a common cause of comorbidities and COPD-related mortality. ${ }^{1-3}$ Invasive ventilation (IV) is essential among AECOPD patients to treat acute respiratory failure. In such cases, strategies should be implemented to discontinue IV as soon as possible to avoid time-dependent complications that may arise as a result of prolonged IV. ${ }^{4}$ Furthermore, extubation failure is associated with a risk of mortality, ranging from 40 to $50 \% .{ }^{5}$

Weaning failure is believed to be multifactorial. However, diaphragmatic dysfunction plays a major role in ventilator dependency, ${ }^{6-8}$ and diaphragmatic function is still poorly assessed among critically ill patients. Although useful for research purposes, direct measurements of diaphragmatic strength are invasive and need considerable expertise. ${ }^{9}$ Diaphragmatic ultrasonography has recently been introduced as a non-invasive and bedside tool to measure diaphragmatic displacement (DD). ${ }^{10,11}$ 
Diaphragmatic dysfunction (defined as DD $<10 \mathrm{~mm}$ ) has been shown to be a good predictor of failure to wean from mechanical ventilation. ${ }^{12}$

The rapid shallow breathing index (RSBI) (defined as the ratio of respiratory rate to tidal volume [RR/VT]) is one of the most widely used predictors of weaning outcome. ${ }^{13}$ But unfortunately, it has variable sensitivity and specificity. ${ }^{14-16}$ So recently, Spadaro et $\mathrm{al}^{17}$ introduced the diaphragmatic RSBI (D-RSBI) by substituting VT with DD in the RSBI (ie, D-RSBI = RR/DD). This avoids masking the underlying diaphragmatic dysfunction caused by the contribution of the accessory muscles in generating VT that could impair the diagnostic accuracy of the RSBI. ${ }^{17}$

The aim of this study was to validate the D-RSBI as a new predictor of weaning outcome in patients with AECOPD and to compare its accuracy with that of the traditional RSBI.

\section{Patients and methods}

\section{Patients}

This prospective observational study was carried out in the Respiratory ICU of the Chest Department, Zagazig University Hospitals during the period from December 2016 to May 2017. It included all patients with AECOPD at their first spontaneous breathing trial (SBT). The exclusion criteria were 1 ) age $<18$ years; 2) presence of thoracostomy, pneumothorax or pneumomediastinum; 3) presence of rib fractures; 4) neuromuscular disease; 5) use of muscle-paralyzing agents within $48 \mathrm{~h}$ before the study and 6) history or new detection of paralysis or paradoxical movement of a single hemidiaphragm on diaphragmatic ultrasonography.

The study was approved by the Faculty of Medicine, Zagazig University Ethics Committee. Written informed consent was obtained from all patients.

\section{Methods}

All studied patients were subjected to the following:

1) A thorough medical history.

2) Full clinical examination (general and local examination).

3) Plain chest X-ray (posteroanterior or anteroposterior view according to circumstances).

4) Routine laboratory investigations:

a) complete blood picture (CBC)

b) liver functions

c) kidney functions

d) serum electrolytes $(\mathrm{Na}, \mathrm{K}, \mathrm{Cl})$.

5) Arterial blood gases analysis (ABGs).
6) Diagnosis of AECOPD according to Global Initiative for Chronic Obstructive Lung Disease (GOLD) criteria: NHLBI/WHO (GOLD) supported by spirometric evidence of airflow obstruction (forced expiratory volume in $1 \mathrm{~s}\left[\mathrm{FEV}_{1}\right] /$ forced vital capacity $[\mathrm{FVC}]<0.70$ ) when clinically stable; with clinical criteria of exacerbation including (increased dyspnea, increased sputum volume or sputum purulence). ${ }^{18}$

7) Calculation of Simplified Acute Physiology Score (SAPS) $\mathrm{II}^{19}$ and assessment of cough strength and amount of endotracheal secretions. ${ }^{20}$

8) Patients intubated and mechanically ventilated for more than $48 \mathrm{~h}$ were considered ready for SBT (ie, spontaneous ventilation through a T-tube with the $\mathrm{FiO}_{2}$ set at the same level used during mechanical ventilation) if they met all of the following criteria: 1) clinical improvement of the underlying acute cause of respiratory failure; 2) favorable cough reflex; 3 ) absence of excessive and/or purulent tracheobronchial secretion; 4) stable cardiovascular status (ie, heart rate $<120$ beats/min; systolic blood pressure, $90-160 \mathrm{mmHg}$; and no vasopressor use); 5) stable metabolic status (ie, electrolytes and blood sugar within normal range, temperature $<38^{\circ} \mathrm{C}$, hemoglobin $\geq 8-10 \mathrm{~g} / \mathrm{dL}$ ); 6) adequate oxygenation (ie, arterial oxygen saturation $(\mathrm{SaO} 2)>92 \%$ with inspiratory oxygen fraction $\left(\mathrm{FiO}_{2}\right) \leq 0.5$ with positive end expiratory pressure $(\mathrm{PEEP}) \leq 8 \mathrm{cmH}_{2} \mathrm{O}$ ) and 7 ) adequate pulmonary function (ie, $\mathrm{RR} \leq 30$ breaths $/ \mathrm{min}$ with $\mathrm{VT} \geq 5 \mathrm{~mL} / \mathrm{kg}$ ideal body weight (IBW) and no significant respiratory acidosis). ${ }^{17}$

9) Ultrasonographic scanning of the right hemidiaphragm after $30 \mathrm{~min}$. from the beginning of the SBT. The patients were lying in the semi recumbent position, with the head of the bed elevated at an angle between $30^{\circ}$ and $45^{\circ}$. Diaphragmatic movement was measured using a $3.5-\mathrm{MHz}$ US probe placed over one of the lower intercostal spaces in the right anterior axillary line with a SonoScape ultrasound machine (SonoScape SSI-4000, SonoScape Medical Corp., Guangdong, China; EC REP SonoScape Europ S.R.L, Rome, Italy). With the probe fixed on the chest wall during respiration, the ultrasound beam was directed to the hemidiaphragmatic dome at an angle of not less than $70^{\circ}$. During inspiration, the normal diaphragm contracts and moves caudally toward the transducer; this is recorded as an upward motion of the M-mode tracing. The amplitude of excursion was measured on the vertical axis of the tracing from the baseline to the point of maximum height of inspiration on the graph. Six measurements were recorded and averaged. All measurements were performed during tidal breathing at $6-12 \mathrm{~mL} / \mathrm{kg}$, excluding smaller or deeper breaths. The whole US examination was accomplished 
in $5 \mathrm{~min}$. Negative excursion was an indicator of paradoxical diaphragmatic movement. ${ }^{12}$ M-mode ultrasonography of the diaphragm of a patient with a conserved right hemidiaphragm function $(\mathrm{DD}=16 \mathrm{~mm})$ is shown in Figure 1. Diaphragmatic ultrasonography was performed for all patients by two independent operators to measure the DD. All patients' recordings were analyzed twice by both operators. All measurements were tested for intraobserver and interobserver reproducibility.

10) Measurement of RR and VT during the SBT using a portable spirometer (Spirotube PC Spirometer, Thor Laboratories, Budapest, Hungary). This PC spirotube has an ultrasonic respiratory sensor with automatic internal calibration.

11) Calculation of RSBI (RR/VT) ${ }^{13}$ and D-RSBI (RR/DD). ${ }^{17}$

12) Assessment of weaning outcome. A successful weaning trial was reported when patients were extubated and breathed spontaneously for more than $48 \mathrm{~h}$. The reinstitution of mechanical ventilation during or at the end of the SBT, reintubation within $48 \mathrm{~h}$ or the use of non-invasive ventilation (NIV) within $48 \mathrm{~h}$ of extubation were reported as a failed weaning trial.

\section{Statistical analysis}

In Spadaro et al's ${ }^{17}$ study, the area under receiver operating characteristic curve (AUROC) for D-RSBI was 0.89 .

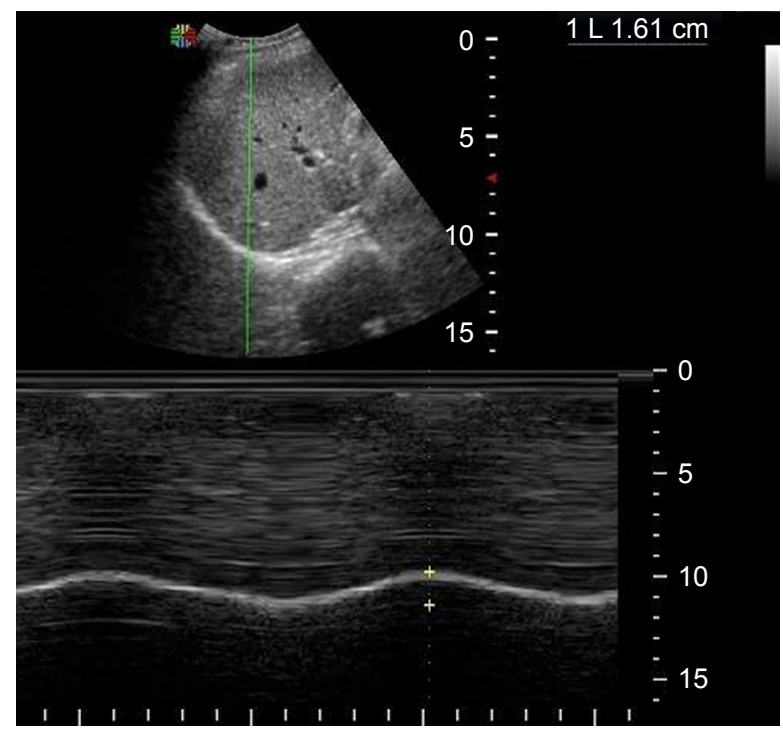

Figure I M-mode ultrasonography of the diaphragm of a patient with a conserved right hemidiaphragm function $(D D=16 \mathrm{~mm})$.

Note: DD was measured on the vertical axis of a frozen image from the baseline, at the end of expiration, to the point of maximum height of inspiration.

Abbreviation: DD, diaphragmatic displacement.
Accordingly, assuming that the null hypothesis for AUROC was 0.5 at a power of $80 \%$, confidence limit of $95 \%$, and a prevalence of $39 \%$ weaning failure in COPD, ${ }^{21}$ a sample size of 41 patients was considered adequate. After estimating a $10 \%$ dropout (refusal or interruption of the measurement), we choose a sample size of 45 patients.

Continuous variables were expressed as the mean $\pm \mathrm{SD}$, and the categorical variables were expressed as a number (percentage). Continuous variables were checked for normality by using the Shapiro-Wilk test. Independent samples Student's $t$-tests were used to compare between two groups of normally distributed variables. Percent of categorical variables were compared using Pearson's chi-square test or Fisher's exact test as appropriate. Receiver operating characteristic (ROC) curve analysis was used to identify optimal cutoff values of DD, RSBI and DSBI with maximum sensitivity and specificity for prediction of weaning failure. The AUROC was also calculated, criteria to qualify for AUC were as follows: $0.90-1=$ excellent, $0.80-0.90=$ good, $0.70-0.80=$ fair, $0.60-0.70=$ poor and $0.50-0.60=$ fail. The optimal cutoff point was established at the point of maximum accuracy. All tests were two sided. A $p$-value $<0.05$ was considered significant. All statistics were performed using SPSS 22.0 for windows (IBM Corporation, Armonk, NY, USA) and MedCalc windows (MedCalc Software bvba 13, Ostend, Belgium).

\section{Results}

The flow chart of the study is illustrated in Figure 2. Initially, 64 patients were screened. A total of 53 of them met the inclusion criteria, while 11 were excluded due to thoracostomy (3 patients), chest wall deformity (1 patient), rib fractures (2 patients) and ventilation for less than $48 \mathrm{~h}$ (5 patients). Additionally, three more patients were excluded because of hemidiaphragm paralysis detected by paradoxical movement of the hemidiaphragm during diaphragmatic ultrasonography. So finally, 50 patients were included and analyzed. Of these, 37 (74\%) were successfully liberated from mechanical ventilation. Among the 13 patients who failed the weaning trial, $8(62 \%)$ failed the SBT and reconnected to the ventilator, $2(15 \%)$ were reintubated within $48 \mathrm{~h}$ of extubation and $3(23 \%)$ required NIV support within $48 \mathrm{~h}$ of extubation.

Table 1 shows no statistically significant differences in preweaning parameters between weaning success and weaning failure groups as regard all demographic and clinical characteristics of the studied patients. 
64 AECOPD patients at their

1st T-piece trial were screened

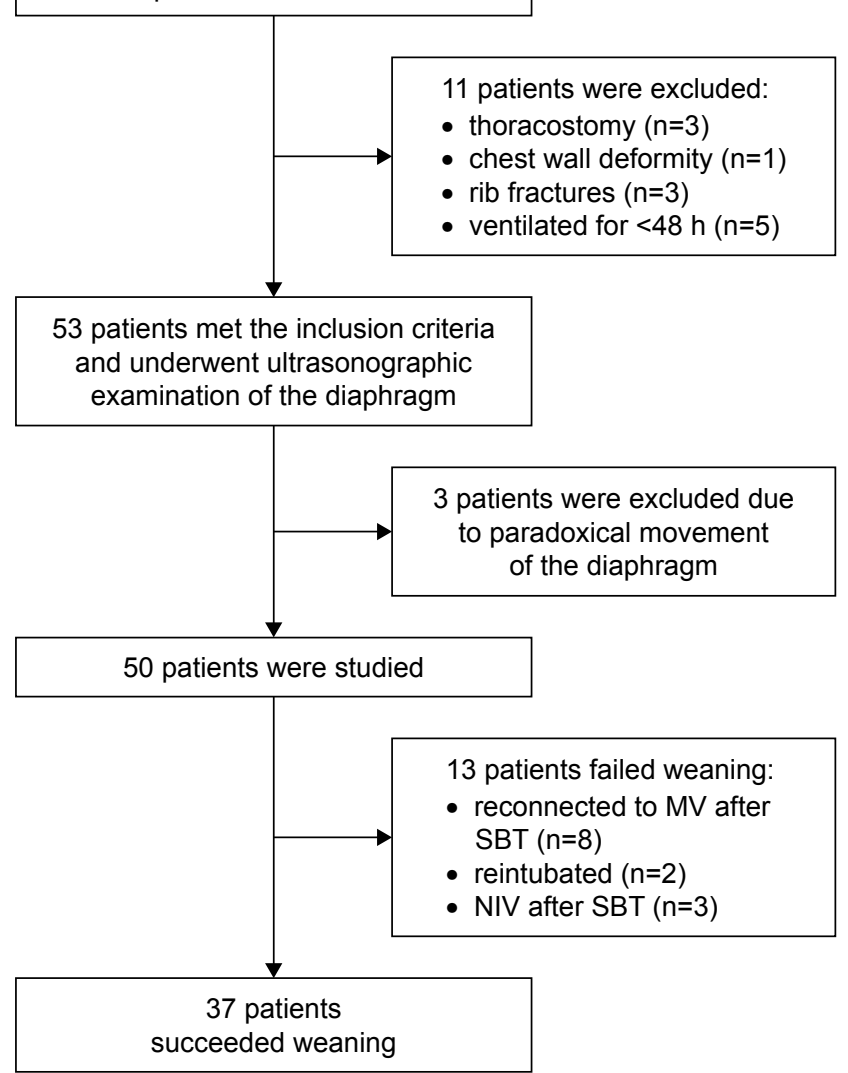

Figure 2 Flow chart of the study.

Abbreviations: AECOPD, acute exacerbation COPD; MV, mechanical ventilation; NIV, non-invasive ventilation; SBT, spontaneous breathing trial.

Table 2 shows the SBT ventilatory parameters among all the studied population. RR, DD and D-RSBI differed significantly between patients who were successfully weaned and those who failed the weaning trial $(p=0.03,<0.001$ and $<0.001$, respectively).

Table 3 and Figure 3 reported the overall results of the ROC analysis regarding the available weaning predictors: D-RSBI, RSBI, DD and RR. D-RSBI showed the best diagnostic accuracy (AUROC $=0.97 ; p<0.001$ ). A cutoff of D-RSBI $>1.9$ breaths $/ \mathrm{min} / \mathrm{mm}$ yielded $84.6 \%$ sensitivity, $100 \%$ specificity, $100 \%$ positive predictive value (PPV), 94.9\% negative predictive value (NPV) and $96 \%$ accuracy.

\section{Discussion}

Mechanical ventilation is a life-saving modality of intervention that is essential for many critically ill patients, including those with AECOPD, but it is associated with a lot of complications as well as patient discomfort. ${ }^{22}$ So, early safe and effective liberation of patients from
Table I Demographic and clinical characteristics of all studied patients

\begin{tabular}{|c|c|c|c|c|}
\hline Variables & $\begin{array}{l}\text { All } \\
(n=50)\end{array}$ & $\begin{array}{l}\text { Weaning } \\
\text { success } \\
(n=37)\end{array}$ & $\begin{array}{l}\text { Weaning } \\
\text { failure } \\
(n=13)\end{array}$ & $p$-value \\
\hline \multicolumn{5}{|l|}{ Sex } \\
\hline Male & $34(68 \%)$ & 27 (79.4\%) & $7(20.6 \%)$ & $0.203^{\ddagger}$ \\
\hline Female & $16(32 \%)$ & $10(62.5 \%)$ & $6(37.5 \%)$ & \\
\hline Age (years) & $61.9 \pm 7.47$ & $61.45 \pm 7.67$ & $63.07 \pm 7.03$ & $0.508 *$ \\
\hline $\mathrm{FEV}_{1}$ & $50.96 \pm 10.7$ & $52.08 \pm 10.6$ & $47.8 \pm 10.4$ & $0.21 *$ \\
\hline FVC & $84.1 \pm 1.6$ & $84.2 \pm 1.6$ & $83.8 \pm 1.7$ & $0.5^{*}$ \\
\hline $\mathrm{FEV} / \mathrm{FVC}$ & $57.9 \pm 4.96$ & $57.7(4.7)$ & $58.5 \pm 5.7$ & $0.6 *$ \\
\hline Disease duration (years) & $8.4 \pm 2.60$ & $8.3 \pm 2.48$ & $8.6 \pm 3.17$ & $0.737^{*}$ \\
\hline \multicolumn{5}{|c|}{ Previous MV } \\
\hline No & $27(54 \%)$ & $21(77.8 \%)$ & $6(22.2 \%)$ & $0.509 \ddagger$ \\
\hline Yes & $23(46 \%)$ & $16(69.6 \%)$ & 7 (30.4\%) & \\
\hline SAPS II on admission & $39.94 \pm 2.87$ & $39.76 \pm 3.06$ & $40.46 \pm 2.29$ & 0.453 \\
\hline \multicolumn{5}{|l|}{ Cough strength } \\
\hline Grade 3 & $13(26 \%)$ & $12(92.3 \%)$ & I (7.7\%) & $0.202^{\ddagger}$ \\
\hline Grade 4 & $20(40 \%)$ & $14(70 \%)$ & $6(30 \%)$ & \\
\hline Grade 5 & 17 (34\%) & II (64.7\%) & $6(35.3 \%)$ & \\
\hline \multicolumn{5}{|l|}{ Amount of secretions } \\
\hline Mild & $28(56 \%)$ & 19 (67.9\%) & $9(32.1 \%)$ & $0.264^{\ddagger}$ \\
\hline Moderate & 22 (44\%) & $18(81.8 \%)$ & $4(18.2 \%)$ & \\
\hline
\end{tabular}

Notes: *Independent samples Student's $t$-test; ${ }^{\ddagger}$ Chi-square test; $p<0.05$ is significant. Continuous variables are expressed as mean \pm standard deviation; categorical variables are expressed as number (percentage).

Abbreviations: $\mathrm{FEV}_{1}$, forced expiratory volume in I s; FVC, forced vital capacity; $\mathrm{MV}$, mechanical ventilation; SAPS, simplified acute physiology score.

mechanical ventilation remains a challenge for clinicians. It is needed, whenever the underlying condition that led to mechanical ventilation has adequately resolved and the patient can breathe spontaneously with sufficient gas exchange. ${ }^{23}$

Two large weaning trials illustrated that clinicians frequently underestimate the ability of patients to breathe spontaneously when liberated from the ventilator. ${ }^{24,25}$ Furthermore, available weaning predictors; like the RSBI lack adequate predictive value to render them routinely used to judge the capability of patients to wean, particularly those at high risk of extubation failure including patients with hypercapnia, COPD or congestive heart failure. ${ }^{25}$ Moreover, due to the complexity of the derangement of the mechanical properties of the respiratory system, COPD patients probably need different weaning indices or different threshold values compared to a general ICU population. $^{26}$

Results of this study confirm the superiority of the diaphragmatic-RSBI (D-RSBI), a new index first adopted by Spadaro and coworkers, ${ }^{17}$ over the traditional RSBI in predicting weaning outcome; even in patients with AECOPD who are at increased risk of extubation failure. 
Table 2 Spontaneous breathing trial ventilatory parameters in all studied patients

\begin{tabular}{|c|c|c|c|c|}
\hline Variables & $\begin{array}{l}\text { All } \\
(n=50)\end{array}$ & $\begin{array}{l}\text { Weaning success } \\
(n=37)\end{array}$ & $\begin{array}{l}\text { Weaning failure } \\
(n=\mid 3)\end{array}$ & $p$-value* \\
\hline RR (breaths/min) & $25.14 \pm 8.27$ & $23.64 \pm 6.48$ & $29.38 \pm 11.27$ & 0.030 \\
\hline $\mathrm{VT}(\mathrm{L})$ & $0.37 \pm 0.14$ & $0.36 \pm 0.12$ & $0.40 \pm 0.20$ & 0.383 \\
\hline $\mathrm{DD}(\mathrm{mm})$ & $|4.66 \pm 4.0|$ & $16.57 \pm 2.4$ & $9.23 \pm 2.42$ & $<0.001$ \\
\hline D-RSBI (breaths/min/mm) & $1.9 \pm 0.96$ & $1.43 \pm 0.32$ & $3.27 \pm 0.84$ & $<0.001$ \\
\hline RSBI (breaths/min/L) & $70.44 \pm 13.76$ & $68.43 \pm 13.59$ & $76.15 \pm 13.10$ & 0.082 \\
\hline
\end{tabular}

Notes: *Independent samples Student's $t$-test; $p<0.05$ is significant. Continuous variables were expressed as mean \pm standard deviation.

Abbreviations: RR, respiratory rate; VT, tidal volume; DD, diaphragmatic displacement; D-RSBI, diaphragmatic rapid shallow breathing index; RSBI, rapid shallow breathing index.

The present work supports the evidence that substitution of VT with DD in calculating RSBI could represent an accurate weaning predictor in a special population of ICU patients, ie, COPD patients with acute exacerbation.

Weaning failure is multifactorial. So, an accurate predictive tool should reflect all pathophysiological determinants of failure, including increased mechanical workload applied on the respiratory muscles, diaphragmatic dysfunction, weaning-induced cardiac failure and a reduced ability to clear secretions and to maintain open airways. Most of the abovementioned pathways lead to rapid shallow breathing. Hence, Yang and Tobin ${ }^{13}$ developed the RSBI which quantitates the extent of rapid shallow breathing taking into account the ratio between RR and VT.

In contrast, patients who failed the weaning attempt in this study had a lower RSBI $(76.15 \pm 13.10$ breaths $/ \mathrm{min} / \mathrm{L})$ than the cutoff value of 105 to predict weaning failure originally described by Yang and Tobin. ${ }^{13}$ Also, several other studies have documented a wide range of predictive values for RSBI, which could reflect differences in methodology, classification of outcomes, and study populations. ${ }^{27,28}$

The present study found that the D-RSBI was significantly higher in COPD patients who failed SBT compared to those who succeeded to wean $(3.27 \pm 0.84$ versus $1.43 \pm 0.32$, respectively), while the traditional RSBI was not significantly different between the two studied groups ( $p=0.082$ ). Also, the D-RSBI predicted weaning failure accurately at a threshold value more than 1.9 (AUROC $=0.97, p<0.001$ ). Although, the cutoff value for the D-RSBI reported by Spadaro and his colleagues ${ }^{17}$ was lower (ie, $>1.3$ ) than this study. This could be explained by the difference in the study population, as the present work included only COPD patients who usually show a lower degree of diaphragmatic excursion. ${ }^{29}$

The D-RSBI was speculated to be more accurate than the RSBI because DD reflects more closely the diaphragmatic function as compared to VT. In the presence of diaphragmatic dysfunction, the diaphragm movement is depressed and the accessory muscles of inspiration assume a greater role in generating VT, but for a limited period of time as they are less efficient and easily fatigable. ${ }^{28,30}$ In these situations, the VT is less linked to the function of the diaphragm than $\mathrm{DD}$, which reflects the ability of the diaphragm to generate inspiratory volume. ${ }^{31}$

Ultrasound examination of the diaphragm is a non-invasive, easy to do and bedside tool that does not require patient collaboration. ${ }^{10,32,33}$ Diaphragmatic M-mode ultrasonography has been recently applied to measure the amount of diaphragmatic excursion (ie, DD) during either spontaneous or assisted

Table 3 Accuracy of DD, D-RSBI, RSBI and RR in predicting weaning failure

\begin{tabular}{|c|c|c|c|c|c|c|c|}
\hline Cutoff values & $\begin{array}{l}\text { Sensitivity \% } \\
(95 \% \text { CI) }\end{array}$ & $\begin{array}{l}\text { Specificity \% } \\
(95 \% \text { CI) }\end{array}$ & $\begin{array}{l}\text { PPV\% } \\
(95 \% \text { CI) }\end{array}$ & $\begin{array}{l}\text { NPV\% } \\
(95 \% \mathrm{CI})\end{array}$ & $\begin{array}{l}\text { Accuracy } \\
(95 \% \mathrm{Cl})\end{array}$ & $\begin{array}{l}\text { AUROC } \\
(95 \% \mathrm{CI})\end{array}$ & $p$-value \\
\hline$D D \leq 12$ & $\begin{array}{l}92.3 \\
(64-99.8)\end{array}$ & $\begin{array}{l}91.9 \\
(78.1-98.3)\end{array}$ & $\begin{array}{l}80 \\
(51.9-95.7)\end{array}$ & $\begin{array}{l}97.1 \\
(84.8-99.9)\end{array}$ & $\begin{array}{l}92 \\
(74.4-98.7)\end{array}$ & $\begin{array}{l}0.975 \\
(0.886-0.999)\end{array}$ & $<0.001$ \\
\hline D-RSBI $>1.9$ & $\begin{array}{l}84.6 \\
(54.6-98.1)\end{array}$ & $\begin{array}{l}100 \\
(90.5-100)\end{array}$ & $\begin{array}{l}100 \\
(71.5-100)\end{array}$ & $\begin{array}{l}94.9 \\
(82.7-99.4)\end{array}$ & $\begin{array}{l}96 \\
(81.2-99.5)\end{array}$ & $\begin{array}{l}0.969 \\
(0.876-0.998)\end{array}$ & $<0.001$ \\
\hline $\mathrm{RSBI}>70$ & $\begin{array}{l}69.2 \\
(38.6-90.9)\end{array}$ & $\begin{array}{l}61.2 \\
(44.8-77.5)\end{array}$ & $\begin{array}{l}39.1 \\
(19.7-61.5)\end{array}$ & $\begin{array}{l}85.2 \\
(66.3-95.8)\end{array}$ & $\begin{array}{l}63.3 \\
(943.2-8 I)\end{array}$ & $\begin{array}{l}0.669 \\
(0.522-0.796)\end{array}$ & 0.065 \\
\hline $\mathrm{RR}>19$ & $\begin{array}{l}100 \\
(75.3-100)\end{array}$ & $\begin{array}{l}29.7 \\
(15.9-47)\end{array}$ & $\begin{array}{l}33.3 \\
(19.1-50.2)\end{array}$ & $\begin{array}{l}100 \\
(71.5-100)\end{array}$ & $\begin{array}{l}48 \\
(31.3-60.8)\end{array}$ & $\begin{array}{l}0.652 \\
(0.504-0.78 I)\end{array}$ & 0.102 \\
\hline
\end{tabular}

Note: $p<0.05$ is significant.

Abbreviations: PPV, positive predictive value; NPV, negative predictive value; AUROC, area under receiver operating characteristic curve; Cl, confidence interval; DD, diaphragmatic displacement; D-RSBI, diaphragmatic rapid shallow breathing index; RSBI, rapid shallow breathing index; RR, respiratory rate. 


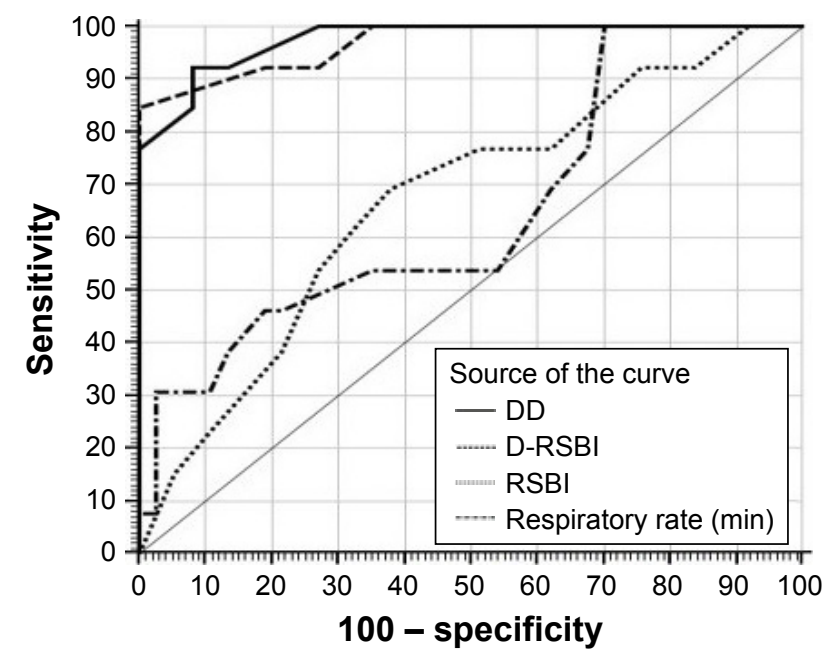

Figure 3 Receiver operating characteristic (ROC) curve for diaphragmatic displacement (DD), diaphragmatic rapid shallow breathing index (D-RSBI), respiratory rate (RR) and traditional rapid shallow breathing index (RSBI).

breathing. ${ }^{11,12,34}$ Herein, the DD was significantly higher in the successfully weaned than in the failed to wean COPD patients. Furthermore, the diagnostic accuracy of DD was superior to that of RSBI but still lower than that of the D-RSBI. These results confirm the superiority of D-RSBI in assessing both diaphragmatic function and prediction of extubation outcome.

Finally, many experts consider that clinical judgment, based upon careful inspection, is superior to and more accurate than available predictors of weaning outcome. ${ }^{24-26}$ However, integrating this clinical judgment with a relatively easy to do bedside index like the D-RSBI may at least help intensivists in their decision to extubate. ${ }^{17}$

Regarding limitations of the current work. First, the reported cutoff value of D-RSBI in the studied COPD patients cannot be generalized to all ICU population and future studies should be done to confirm the validity of the D-RSBI in predicting the weaning outcome in different clinical situations. Second, one can say that ultrasonography is an operator dependent and measurements of DD might change between operators, but in the current work, interobserver and intraobserver reproducibility were tested.

\section{Conclusion}

D-RSBI (RR/DD) is a new and promising tool that is superior to the traditional RSBI (RR/VT) in predicting weaning outcome in COPD patients with acute exacerbation.

\section{Acknowledgment}

We wish to thank all the medical staff on the respiratory ICU, Zagazig University, Egypt.

\section{Author contributions}

$\mathrm{AA}$ and SE were responsible for the study design. AA and MW were responsible for patient selection, data collection and performing diaphragmatic ultrasonography for all selected patients according to the adopted protocol.

All authors contributed toward data analysis, drafting and revising the paper and agree to be accountable for all aspects of the work.

\section{Disclosure}

The authors report no conflicts of interest in this work.

\section{References}

1. Higgins MW, Thom T. Incidence, prevalence and mortality: intra- and inter-country difference. In: Hensley MJ, Saunders NA, editors. Clinical Epidemiology of Chronic Obstructive Pulmonary Disease. New York: Marcel Dekker; 1990:23-43.

2. Connors AF Jr, Dawson NV, Thomas C, et al. Outcomes following acute exacerbation of severe chronic obstructive lung disease. The SUPPORT investigators (Study to Understand Prognoses and Preferences for Outcomes and Risks of Treatment). Am J Respir Crit Care Med. 1996;154(4 Pt 1):959-967.

3. Fuso L, Incalzi RA, Pistelli R, et al. Predicting mortality of patients hospitalized for acutely exacerbated chronic obstructive pulmonary disease. Am J Med. 1995;98(3):272-277.

4. Song Y, Chen R, Zhan Q, et al. The optimum timing to wean invasive ventilation for patients with AECOPD or COPD with pulmonary infection. Int J Chron Obstruct Pulmon Dis. 2016;11:535-542.

5. Thille AW, Harrois A, Schortgen F, Brun-Buisson C, Brochard L. Outcomes of extubation failure in medical intensive care unit patients. Crit Care Med. 2011;39(12):2612-2618.

6. McCool FD, Tzelepis GE. Dysfunction of the diaphragm. $N$ Engl $J$ Med. 2012;366(10):932-942.

7. Jaber S, Petrof BJ, Jung B, et al. Rapidly progressive diaphragmatic weakness and injury during mechanical ventilation in humans. $\mathrm{Am} J$ Respir Crit Care Med. 2011;183(3):364-371.

8. Hermans G, Agten A, Testelmans D, Decramer M, Gayan-Ramirez G. Increased duration of mechanical ventilation is associated with decreased diaphragmatic force: a prospective observational study. Crit Care. 2010;14(4):R127.

9. Doorduin J, van Hees HW, van der Hoeven JG, Heunks LM. Monitoring of the respiratory muscles in the critically ill. Am J Respir Crit Care Med. 2013;187(1):20-27.

10. Matamis D, Soilemezi E, Tsagourias M, et al. Sonographic evaluation of the diaphragm in critically ill patients. Technique and clinical applications. Intensive Care Med. 2013;39(5):801-810.

11. Boussuges A, Gole Y, Blanc P. Diaphragmatic motion studied by M-mode ultrasonography. Chest. 2009;135(2):391-400.

12. Kim WY, Suh HJ, Hong SB, Koh Y, Lim CM. Diaphragm dysfunction assessed by ultrasonography: influence on weaning from mechanical ventilation. Crit Care Med. 2011;39(12):2627-2630.

13. Yang KL, Tobin MJ. A prospective study of indexes predicting the outcome of trials of weaning from mechanical ventilation. $N$ Engl $J$ Med. 1991;324(21):1445-1450.

14. Lee KH, Hui KP, Chan TB, Tan WC, Lim TK. Rapid shallow breathing (frequency tidal volume ratio) did not predict extubation outcome. Chest. 1994;105(2):540-543.

15. Savi A, Teixeira C, Silva JM, et al; for the Gaúcho Weaning Study Group. Weaning predictors do not predict extubation failure in simpleto-wean patients. J Crit Care. 2012;27(2):221.e1-e8. 
16. Huang CT, Yu CJ. Conventional weaning parameters do not predict extubation outcome in intubated subjects requiring prolonged mechanical ventilation. Respir Care. 2013;58(8):1307-1314.

17. Spadaro S, Grasso S, Mauri T, et al. Can diaphragmatic ultrasonography performed during the $\mathrm{T}$-tube trial predict weaning failure? The role of diaphragmatic rapid shallow breathing index. Crit Care. 2016;20(1):305.

18. Global Initiative for Chronic Obstructive Lung Disease (GOLD) for the diagnosis, management strategy and prevention of chronic obstructive lung disease (updated 2016) [homepage on the Internet]. Available at: http://www.goldcopd.com. Accessed December 18, 2016.

19. Le Gall JR, Lemeshow S, Saulnier F. A new Simplified Acute Physiology Score (SAPS II) based on a European/North American multicenter study. JAMA. 1993;270(24):2957-2963.

20. Khamiees M, Raju P, DeGirolamo A, Amoateng-Adjepong Y, Manthous CA. Predictors of extubation outcome in patients who have successfully completed a spontaneous breathing trial. Chest. 2001;120(4):1262-1270.

21. Abu Youssef HA, Shalaby AEO, Abd El Hafiz AM, Shaban MM, Hamed HAG. Predictive value of rapid shallow breathing index in relation to the weaning outcome in ICU patients. Egypt $J$ Chest Dis Tuberculosis. 2016;65(2):465-472.

22. Schmidt GA, Girard TD, Kress JP, et al. Liberation from mechanical ventilation in critically ill adults. Executive Summary of an Official American College of Chest Physicians/American Thoracic Society Clinical Practice Guideline. Chest. 2017;151(1):160-165.

23. Ouellette DR, Patel S, Girard TD, et al. Liberation from mechanical ventilation in critically ill adults: an Official American College of Chest Physicians/American Thoracic Society Clinical Practice Guideline. Inspiratory pressure augmentation during spontaneous breathing trials, protocols minimizing sedation, and noninvasive ventilation immediately after extubation. Chest. 2017;151(1):166-180.

24. Brochard L, Rauss A, Benito S, et al. Comparison of three methods of gradual withdrawal from ventilatory support during weaning from mechanical ventilation. Am J Respir Crit Care Med. 1994;150(4): 896-903.
25. Esteban A, Frutos F, Tobin MJ, et al. A comparison of four methods of weaning patients from mechanical ventilation. Spanish Lung Failure Collaborative Group. N Engl J Med. 1995;332(6):345-350.

26. Alvisi R, Volta CA, Righini ER, et al. Predictors of weaning outcome in chronic obstructive pulmonary disease patients. Eur Respir J. 2000;15(4): 656-662.

27. Vallverdu I, Calaf N, Subirana M, Net A, Benito S, Mancebo J. Clinical characteristics, respiratory functional parameters, and outcome of a two hour T-piece trial in patients weaning from mechanical ventilation. Am J Respir Crit Care Med. 1998;158(6):1855-1862.

28. Yan S, Lichros I, Zakynthinos S, Macklem PT. Effect of diaphragmatic fatigue on control of respiratory muscles and ventilation during $\mathrm{CO}_{2}$ rebreathing. J Appl Physiol. 1993;75(3):1364-1370.

29. Unal O, Arslan H, Uzun K, Ozbay B, Sakarya ME. Evaluation of diaphragmatic movement with MR fluoroscopy in chronic obstructive pulmonary disease. Clin Imaging. 2000;24(6):347-350.

30. Yan S, Sliwinski P, Gauthier AP, Lichros I, Zakynthinos S, Macklem PT. Effect of global inspiratory muscle fatigue on ventilatory and respiratory muscle responses to CO2. J Appl Physiol. 1993;75(3): 1371-1377.

31. Umbrello M, Formenti P, Longhi D, et al. Diaphragm ultrasound as indicator of respiratory effort in critically ill patients undergoing assisted mechanical ventilation: a pilot clinical study. Crit Care. 2015; 19:161.

32. Goligher EC, Laghi F, Detsky ME, et al. Measuring diaphragm thickness with ultrasound in mechanically ventilated patients: feasibility, reproducibility and validity. Intensive Care Med. 2015;41(4):642-649.

33. Testa A, Soldati G, Giannuzzi R, Berardi S, Portale G, Gentiloni Silveri N. Ultrasound M-mode assessment of diaphragmatic kinetics by anterior transverse scanning in healthy subjects. Ultrasound Med Biol. 2011;37(1):44-52.

34. Demoule A, Jung B, Prodanovic H, et al. Diaphragm dysfunction on admission to the intensive care unit. Prevalence, risk factors, and prognostic impact - a prospective study. Am J Respir Crit Care Med. 2013 188(2):213-219.
International Journal of COPD

\section{Publish your work in this journal}

The International Journal of COPD is an international, peer-reviewed journal of therapeutics and pharmacology focusing on concise rapid reporting of clinical studies and reviews in COPD. Special focus is given to the pathophysiological processes underlying the disease, intervention programs, patient focused education, and self management protocols.

\section{Dovepress}

This journal is indexed on PubMed Central, MedLine and CAS. The manuscript management system is completely online and includes a very quick and fair peer-review system, which is all easy to use. Visit http://www.dovepress.com/testimonials.php to read real quotes from published authors. 